\title{
Aportaciones femeninas a las teorías de la comunicación: una propuesta para la docencia y la ciencia
}

\author{
Leonarda García-Jiménez \\ Universidad de Murcia \\ Colorado State University \\ leonardagj@um.es
}

Fecha de presentación: junio de 2020

Fecha de aceptación: abril de 2021

Fecha de publicación: diciembre de 2021

Cita recomendada: GARCÍA-JIMÉNEZ, L. (2021). «Aportaciones femeninas a las teorías de la comunicación: Una propuesta para la docencia y la ciencia». Anàlisi: Quaderns de Comunicació i Cultura, 65, 121-135. DOI: <https://doi.org/10.5565/rev/analisi.3327>

\section{Resumen}

Las teorías de la comunicación son un campo de estudio en el que con frecuencia se han silenciado las aportaciones de mujeres investigadoras. Por ello, este artículo tiene como principal objetivo desarrollar un marco que analice el campo de la comunicación a partir de la figura de destacadas investigadoras que históricamente han tendido a quedar al margen de los relatos oficiales a pesar de la importancia de sus contribuciones. A partir de la revisión crítica de textos históricos y de referencia, el artículo propone una genealogía de destacadas investigadoras de la primera generación (delimitada entre las décadas de 1930 y 1960) dentro de los enfoques dominantes de las teorías de la comunicación: funcionalista, interpretativo y crítico. Como resultado, esta aproximación hermenéutica y crítica destaca el contexto intelectual de Columbia como epicentro del pensamiento femenino a mediados del siglo XX y recupera a figuras clave, también denominadas «madres fundadoras», en el establecimiento del campo de la comunicación, como fueron: Herta Herzog, Hazel Gaudet, Thelma Anderson, Marjorie Fiske, Hortense Powdermarker, Else Frenkel-Brunswik, Mae Huettig, Helen Hughes, Rachel Powell y Mary Q. Innis. Esta propuesta abre y hace más heterogéneo el canon oficial de las teorías de la comunicación, dominado históricamente por figuras masculinas. A modo de conclusión, el artículo plantea la necesidad de incorporar al campo los

* Este artículo forma parte del proyecto «Feminizar las ciencias de la comunicación: memoria y aportaciones de mujeres investigadoras", realizado con una Beca Leonardo a Investigadores y Creadores Culturales 2019 de la Fundación BBVA. La Fundación BBVA no se responsabiliza de las opiniones, comentarios y contenidos incluidos en el proyecto y/o resultados derivados del mismo, los cuales son total y absoluta responsabilidad de la autora. 
aportes femeninos, una cuestión que ayudará a superar el sesgo de género con el que todavía se enseñan y se piensan hoy las teorías de la comunicación.

Palabras clave: teorías de la comunicación; mujeres investigadoras; epistemología crítica; investigación; docencia; género

Resum. Aportacions femenines a les teories de la comunicació: una proposta per a la docència i la ciència

Les teories de la comunicació són un camp d'estudi en el qual sovint s'han silenciat les aportacions de les dones investigadores. Per això, aquest article té com a objectiu principal desenvolupar un marc que analitzi el camp de la comunicació a partir de la figura de destacades investigadores que històricament han tendit a quedar al marge dels relats oficials tot i la importància de les seves contribucions. A partir de la revisió crítica de textos històrics i de referència, l'article proposa una genealogia de destacades investigadores de la primera generació (delimitada entre les dècades de 1930 i 1960) dins dels enfocaments dominants de les teories de la comunicació: funcionalista, interpretatiu i crític. Com a resultat, aquesta aproximació hermenèutica i crítica destaca el context intel-lectual de Columbia com a epicentre del pensament comunicatiu femení a mitjan segle XX i recupera algunes figures clau en la fundació del camp de la comunicació: Herta Herzog, Hazel Gaudet, Thelma Anderson, Marjorie Fiske, Hortense Powdermarker, Else Frenkel-Braunschweig, Mae Huettig, Helen Hughes, Rachel Powell i Mary Q. Innis. Aquesta proposta obre i fa més heterogeni el cànon oficial de les teories de la comunicació, dominat històricament per figures masculines. A tall de conclusió, l'article planteja la necessitat d'incorporar al camp les aportacions femenines, una qüestió que ajudarà a superar el biaix de gènere a partir del qual encara s'ensenyen i es pensen les teories de la comunicació en l'actualitat.

Paraules clau: teories de la comunicació; dones investigadores; epistemologia crítica; investigació; docència; gènere

\section{Abstract. Female contributions to communication theories: a teaching and scientific proposal}

Communication theories are a field of study in which the scientific contributions of female researchers have tended to be silenced. For this reason, the main purpose of this paper is to develop a framework to analyse the field of communication based on leading researchers who historically have tended to remain at the margins of official accounts, despite the importance of their contributions. Starting with a critical review of historical publications and key texts, this paper proposes a genealogy of female researchers from the first generation (1930s-1960s) within the main traditions of communication theories: functionalist, interpretive and critical. As a result, this hermeneutical and critical approach highlights the intellectual context of Columbia as the epicentre of female thought in the mid-twentieth century. Furthermore, the paper recovers certain key figures in the founding of the field of communication, including: Herta Herzog, Hazel Gaudet, Thelma Anderson, Marjorie Fiske, Hortense Powdermarker, Else Frenkel-Brunswik, Mae Huettig, Helen Hughes, Rachel Powell and Mary Q. Innis. This proposal opens up and makes more heterogeneous the official canon of communication theories, historically dominated by male researchers. This paper concludes with the need to include female contributions in the field of communication. This will help overcome the gender bias that still characterises teaching and research in communication theory.

Keywords: communication theories; female researchers; critical epistemology; research; teaching; gender 


\section{Introducción y estado de la cuestión}

Un día, unas cuantas de nosotras estábamos trabajando en uno de los despachos que el gobierno estadounidense había habilitado para llevar a cabo la investigación de la Mass Communication Research. Paul Lazarsfeld entró en la sala, miró alrededor y dijo: «OK, podemos entrar, aquí no hay nadie». «No había nadie», pero estábamos cuatro o cinco de nosotras trabajando, codificando, haciendo tablas, sacando porcentajes... "Aquí no hay nadie», porque eso éramos nosotras: «Nadie».

Así comienza el documental Out of the Question sobre el papel que mujeres investigadoras jugaron en la configuración de la investigación en comunicación durante el período de entreguerras. Relata la anécdota Joan Goldhamer, colaboradora en las investigaciones funcionalistas de la Mass Communication Research (Simonson y Archer, 2011), quien hace referencia a Paul Lazarsfeld, pionero en este campo de estudio y en las teorías de los efectos de los medios. En este momento histórico se fraguó el análisis empírico de la comunicación y los medios y en él se organizó la disciplina de forma institucional tal y como la entendemos hoy en día (Rowland y Simonson, 2013: 5).

La anécdota es tremendamente significativa y funciona como una potente metáfora representativa de la escasísima visibilidad que las mujeres investigadoras han tenido dentro de la disciplina de la comunicación (Signorielli, 1996; Simonson y Archer, 2011; Vera Balanza, 2012; Dorsten, 2012 y 2016; Rowland y Simonson, 2013; García-Jiménez, 2019). Vivieron la fundación de la especialidad a mediados del siglo XX como investigadoras principales y autoras de teorías y métodos científicos, secretarias, al frente de los trabajos de campo, etc. (Rowland y Simonson, 2013; Simonson y Archer, 2011), pero su impronta se pierde con el paso de los años.

Una potente borradura de la figura femenina es la historia (Park y Pooley, 2008) que escribió Wilbur Schramm (1963) en los años 60 con el mito sobre los "padres fundadores", que retoma más adelante Everett Rogers (1994) para consolidarlo en nuestro imaginario colectivo. Cuando miramos a la historia de los estudios en comunicación e incluso cuando enseñamos teorías de la comunicación, recurrimos con frecuencia a esta leyenda (Park y Pooley, 2008: 3; Vorderer, Park y Lutz, 2019: 3; Pooley, 2008 y 2017; Rowland y Simonson, 2013), que señala que los pioneros y creadores de nuestro campo de estudio fueron Paul Lazarsfeld, Kurt Lewin, Carl Hovland y Harold Lasswell. Este mito simplifica la comprensión del campo y se origina en la historia que el propio Schramm publica por primera vez en el capítulo "Communication research in the United States», incluido en el libro que él mismo edita en 1963, The science of human communication. Este capítulo es el «desvergonzado origen del mito. No es histórico, ni es sistemático, sino una caricatura para dar legitimidad» (Pooley, 2008: 46). El texto de Schramm, que a la postre también fue reconocido como padre fundador, perfiló el canon y delimitó el campo de conocimiento, haciendo muy complejo salir de este marco y reconocer otras perspectivas y autorías. Recordemos que las teorías 
no son sino una manera de ver y pensar el mundo (Deetz, en García-Jiménez, 2019: 38-39).

Como vemos, el mito ha ayudado a sostener y reproducir la figura masculina como paradigma de creación científica (los "padres fundadores») y construcción disciplinaria, aunque, como recuerda Dorsten (2016: 3): «Las mujeres investigadoras estuvieron en los mismos círculos. Si rascamos y vamos más allá del mito, encontramos mujeres». Pareciera que la narrativa llegó para quedarse, si bien recientes aproximaciones históricas la han empezado, poco a poco, a denunciar y deconstruir (Park y Pooley, 2008; Pooley, 2017; Simonson y Park, 2015; Vorderer, Park y Lutz, 2019).

De esta forma, los relatos que han conformado las ciencias y las teorías de la comunicación han estado dominados por investigadores. Si atendemos al enfoque clásico de las teorías de la comunicación, aquel que parte de las perspectivas y escuelas (Rodrigo Alsina, 2001; Craig, 1999), veremos que entre los autores que inician la investigación en comunicación desde diferentes enfoques destacan, principalmente, nombres masculinos (Adorno, Horkheimer, Marcuse, Benjamin, Habermas, Hoggart, Hall, Thompson, Berger y Luckmann, Lippmann, Mead, Cooley, Park, Blumer, Goffman, Lasswell, Lazarsfeld, Hovland, Lewin, Merton, etc.). En el canon se cuelan unos pocos nombres femeninos de esta primera generación, delimitada entre las décadas de 1930 y 1960 (Dorsten, 2016: 1), principalmente Gladys Lang y Elizabeth Noelle-Neumann (Vera Balanza, 2012: 17). El resto de autoras que aparecen en algunos pocos textos docentes de teorías pertenecerían ya a la segunda generación (de la década de 1960 a la de 1990), en la que se incluiría a Gaye Tuchman, Pamela Shoemaker, Ien Ang, Tania Modlesky, Janice Radway, Sandra Ball-Rokeach, Liesbet van Zoonen o Michelle Mattelart (Vera Balanza, 2012: 18).

Por todo ello, el propósito del presente texto es recuperar la memoria de destacadas investigadoras de la primera generación que funda las principales escuelas de pensamiento y resignificar, con ello, el rol que la mujer ha desempeñado en la disciplina de la comunicación en su vertiente más teórica. En última instancia, esta investigación plantea el desarrollo de una mirada de género sobre las teorías de la comunicación, perspectiva que, en general, está ausente de los planes de estudio de las facultades de comunicación en España (García, Zurián y Núñez, 2020). Debemos re-imaginar y re-diseñar la forma en la que hemos contado la historia de la disciplina y la forma en la que hemos enseñado y enseñamos todavía hoy teorías e investigación en comunicación, de ahí la mirada y el mapa femenino sobre nuestros estudios que planteo en el marco de este trabajo.

\section{Metodología: una propuesta para reconstruir el campo de la comunicación}

Para tratar de salir del marco patriarcal y excluyente que he presentado en el apartado anterior, resulta de suma utilidad el entender a las escuelas de pensa- 
miento mediante la teoría del ensamblaje (Rowland y Simonson, 2013: 7), que implica concebirlas como «una colección heterogénea e históricamente particular de partes cuyas interacciones y agencias dispersas producen una gama de efectos emergentes». De esta forma, concebir a las escuelas de pensamiento no como espacios estáticos de producción de conocimiento, sino más bien como redes de interacción (profesionales y personales), permite realizar una aproximación más fidedigna a la comprensión de los procesos de investigación que tuvieron lugar entre las décadas de 1930 y 1960 y que dieron como resultado algunas de las aportaciones más importantes en el ámbito de las teorías de la comunicación, en las que con frecuencia participaron mujeres investigadoras.

A partir de este enfoque, que permite entender a las escuelas y a las perspectivas de manera más flexible a como hemos hecho hasta ahora, la metodología que ha seguido este trabajo se articula a partir de una amplia revisión crítico-hermenéutica de textos históricos y de referencia en el marco epistemológico de este artículo. Concretamente, se han revisado:

1. Trabajos metateóricos que han recuperado las aportaciones de destacadas académicas en comunicación y en otras disciplinas afines (Dorsten, 2012 y 2016; Rowland y Simonson, 2013; Simonson, 2012; Striphas y Hayward, 2013; Hier y Kemp, 2002; Black, 2003; Connel y Hilton, 2015; Belisle y Mitchell, 2018; Klaus y Seethaler, 2017; Commire y Klezmer, 2007; Phillips, 2015).

2. Publicaciones históricas de las investigadoras aparecidas en la revisión metateórica (Frenkel-Brunswik, 1939, 1940, 1948 y 1949; Huettig, 1944; Hughes, 1940 y 1973; Powell, 1965; Innis, 1935).

3. Aproximaciones a la historia intelectual de las ciencias sociales (Fleck, 2011; Valsiner y Abbey, 2006; Creighton, 1957).

A partir de esta triple revisión crítico-hermenéutica planteo el siguiente mapa de destacadas investigadoras de la primera generación dentro de los principales paradigmas de las teorías de la comunicación (Rodrigo Alsina, 2001). También señalo el lugar y el año de nacimiento, la fecha de fallecimiento y/o los matrimonios y la dirección doctoral, en caso de que esta información fuera significativa para entender los diferentes «ensamblajes teóricos» de nuestra disciplina:

- Enfoque sociopsicológico-funcionalista-empírico-deductivo:

- Ensamblaje de la Escuela de Columbia: Herta Herzog (Austria, 1910-2010, casada en primeras nupcias y divorciada de Paul Lazarsfeld, quien también le dirige la tesis doctoral); Helen Lynd (EE. UU., 1896-1982, casada con Robert Lynd); Ann Miller (EE.UU., 19212006, discípula de Dorothy Thomas); Marjorie Fiske (EE. UU., 1914-1992, esposa en segundas nupcias de Leo Löwenthal, de quien se terminó divorciando); Hazel Gaudet (EE.UU., 1908-1975); Rose K. Goldsen (EE.UU., 1917-1985); Gladys Lang (EE.UU., 1919- 
2016, casada con Kurt Lang); Thelma Ehrlich Anderson (EE. UU., 1921-2012, estudiante de Paul Lazarsfeld), y Joan Doris Goldhamer (EE. UU., 1922, casada con Herbert Goldhamer).

- Desde una perspectiva sociopsicológico-positivista más amplia: Hilde Himmelweit (Alemania, 1918-1989).

- Desde la perspectiva de la opinión pública: Elizabeth Noelle-Neumann (Alemania, 1916-2010).

- Ensamblaje de la Escuela de Toronto: Mary Q. Innis (EE. UU., 1899-1972, casada con Harold Innis).

- Enfoque interpretativo-sociocultural-fenomenológico-empírico-inductivo:

- Helen MacHill Hughes (EE. UU., 1903-1992, casada con Everett Hughes y discípula de Robert E. Park), y Dorothy S. Thomas (EE. UU., 1899-1977, casada con William Thomas, directora de tesis de Ann Miller) (ensamblaje de la Escuela de Chicago).

- Desde una perspectiva interpretativa más amplia, Hortense Powdermarker (EE. UU., 1896-1970, discípula de Edward Sapir, Bronisław Malinowski y Radclife Brown); Ruth Benedict (EE. UU., 1887-1948), directora de tesis de Margaret Mead (EE. UU., 1901-1978, casada con Gregory Bateson) con quien mantuvo una relación romántica.

- Enfoque crítico-cultural-psicoanalítico:

- Dentro del ensamblaje de la Escuela de Frankfurt: Else FrenkelBrunswik (Lvov, Imperio Austrohúngaro, 1908-1958).

- Desde una perspectiva psiconalítica más amplia: Karen Horney (Alemania, 1885-1952).

- Hannah Arendt en teoría política (Alemania, 1906-1975).

- Mae D. Huettig (EE. UU., 1911-1996) desde la perspectiva de la economía política de la comunicación.

- Rachel Powell dentro del ensamblaje de la Escuela de Birmingham.

Desarrollo a continuación algunas de las figuras más significativas de este mapa conceptual.

\section{Resultados: aportaciones de investigadoras a la disciplina comunicativa}

\subsection{Columbia como epicentro femenino de la producción cientifica}

Desde una perspectiva de género, merece especial atención la Mass Communication Research (enfoque sociopsicológico-funcionalista-empírico-deductivo) por el destacado rol que tuvieron las investigadoras durante la Segunda Guerra Mundial. En principio, no habría ninguna otra escuela en comunicación que durante las décadas de 1930 a 1960 albergara el potencial femenino de la Office of Radio Research (ORR, en el marco de la Universidad de Princeton), primero, y su sustituta la Bureau of Applied Social Research (conocida también como la Bureau, a secas, dependiente de la Universidad de Columbia). 
Las investigadoras tuvieron una importante presencia sobre todo en el periodo que va desde 1937 hasta 1945, momento en el que hasta 34 mujeres fueron autoras o coautoras de trabajos (Rowland y Simonson, 2013: 9). El ensamblaje de la conocida como Escuela de Columbia fue un contexto privilegiado para el crecimiento profesional y científico de las investigadoras, teniendo en cuenta, además, que los estudios de recepción y audiencias estadounidenses más importantes de la época (Personal influence, Mass persuasion u On borrowed experience) se hicieron mediante entrevistas y grupos focales con sujetos informantes principalmente femeninos (Douglas, 2006; Simonson, 2012). Por ello, la figura de la mujer adquirió una doble importancia: como investigadora y a la vez como objeto de estudio. Es el caso de Personal influence, publicación que plantea la teoría de efectos limitados y el two step flow, que contó con la participación de 800 mujeres, sujetos informantes a los que con frecuencia la obra se refiere como respondents, influential persons, consumers o he para referirse al líder (Douglas, 2006: 42). Este uso lingüístico enmascaró el rol decisivo protagonizado por las audiencias femeninas, lo que, paradójicamente, a la postre supuso el tomar a la mujer como representativa «en la sombra» del todo social.

Los estudios de fan a través de las cartas enviadas por correo postal ( $\mathrm{fan}$ mail studies) llevados a cabo por Jeannette Sayre, Rowena Wyant y Herta Herzog también estuvieron centrados en audiencias exclusivamente femeninas (Simonson, 2012: 1282), aunque estas investigaciones, al igual que ha sucedido con otras muchas, fueron borradas del mapa, en parte, a partir de la tesis doctoral sobre fan mail studies de Elihu Katz (Simonson, 2012: 1283).

El cambio en el protagonismo de las investigadoras se produjo con la vuelta de los hombres del frente bélico europeo, que tuvieron prioridad para incorporarse al mundo laboral (Douglas, 2006: 44) y, ya a partir de 1948, «más y más hombres fueron contratados y las mujeres encontraron pocas oportunidades en el campo de la comunicación" (Simonson, 2012: 1281). La presencia femenina en comunicación quedaría eclipsada en Estados Unidos hasta finales de la década de 1960, cuando las mujeres empezaron a «saltar la valla» (Dorsten, 2016: 3), incorporándose a la universidad con figuras estables.

En este contexto merece especial mención Herta Herzog, pionera en la investigación de audiencias y adelantada a su tiempo por el planteamiento del paradigma de la audiencia activa. Ella fue una de las primeras en tomar los géneros menores, en su caso las telenovelas radiofónicas, como objetos de estudio relevantes, un giro hacia formas culturales consideradas de segunda clase que décadas más tarde también encontraremos en los estudios culturales británicos (Brunsdon, 2000). Herzog abordó la complejidad de la experiencia mediática (Klaus y Seethaler, 2016) y cuenta en su haber con importantes hitos sin los cuales no sería posible entender hoy la investigación, con el desarrollo del paradigma de los usos y las gratificaciones y con sus aportes metodológicos. Con respecto a estos últimos, es preciso destacar sus contribuciones a las metodologías cualitativas ampliamente utilizadas en la contemporaneidad, como la entrevista en profundidad o la invención del 
focus group, aunque se haya adjudicado la creación de este último de manera injusta a Robert K. Merton, a pesar de que Herzog lo utilizó, también las entrevistas, varios años antes que él (Rowland y Simonson, 2013: 11). Todas estas cuestiones hacen de esta autora un referente que solamente de forma más reciente empieza a ser recuperado (Klaus y Seethaler, 2016).

También destacan en este contexto Thelma Anderson, responsable de la realización de entrevistas y cuya contribución fue decisiva (Simonson y Archer, 2011) en obras como Personal influence (Katz y Lazarsfeld, 1955), o Voting (Berelson, Lazarsfeld y McPhee, 1954); Hazel Gaudet, coautora de The invasion from mars (Cantril, Gaudet y Herzog, 1940) y The people's choice (Lazarsfeld, Berelson y Gaudet, 1944), o Marjorie Fiske, coautora de Mass persuasion (Merton, Fiske y Curtis, 1946) y de The focused interview (Merton, Fiske y Kendall, 1948), entre otras muchas publicaciones que podrían ser enmarcadas en el campo de la comunicación y los medios.

Que en la mayoría de las ocasiones estas coautorías hayan caído en el olvido responde a una construcción del conocimiento científico marcada por el sesgo de género, que ha sido un "constituyente crucial para el análisis de las relaciones de poder de todos los campos y en todas las disciplinas y estructuras de conocimiento" (Jansen, 1993: 142). Precisamente por ello, The invasion from mars se suele adjudicar exclusivamente a Cantril; Mass persuasion, a Merton; The people's choice, a Lazarsfeld; etc. En algunas de estas publicaciones se rebajaba la autoría de las investigadoras con el eufemismo with the assistance of ('con la colaboración de'), como sucede en las primeras ediciones de The invasion from mars o The people's choice.

Finalmente, Hortense Powdermarker, de impronta cualitativa y etnográfica, también podría ser enmarcada en el ensamblaje de Columbia a pesar de su orientación antropológica, una cuestión que subraya que el contexto de la Mass Communication Research fue más interdisciplinario que la imagen positivista con la que se le ha asociado, al menos durante el periodo de intervención femenina comprendido entre 1937 y 1945 . Powdermarker desarrolló la importancia de la etnografía en el análisis de la comunicación de masas y cómo las audiencias se identifican con los textos mediáticos. Ella es otro referente olvidado, una autora que trabajó y se formó con el antropólogo Edward Sapir y los funcionalistas Bronisław Malinowski y Radclife Brown. $\mathrm{Su}$ aproximación interdisciplinaria a entender la comunicación de masas la hizo una afamada crítica del imperialismo conceptual funcionalista que empezaba a imponerse en la década de 1950 (no antes) (Dorsten, 2012). Reputada antropóloga, fue la primera mujer que investigó sola en el Pacífico sur y ha sido considerada una científica ecléctica, dinámica y avanzada a su tiempo (Hier y Kemp, 2002). Se caracterizó por plantear una agenda investigadora ambiciosa, con cuestiones relacionadas con la raza, el racismo, el poder, el conflicto o la despersonalización e interesada por las formas culturales de las sociedades, una cuestión que la llevó a la comunicación de masas, concretamente, al análisis antropológico y crítico de la industria cinematográfica de Hollywood (Hier y Kemp, 2002). Recoge Dorsten (2012: 41) que sus 
experiencias académicas fueron suficientemente opresivas para que acabara con una depresión, en parte producida por el aislamiento que vivieron las investigadoras en la universidad y que, como veremos en el siguiente apartado, también denunció Helen Hughes (1973).

\subsection{De la Escuela de Frankfurt al determinismo tecnológico}

Pero Columbia no es el único contexto en el que es posible destacar las aportaciones femeninas. Los ensamblajes de las Escuelas de Frankfurt, economía política, Chicago, estudios culturales y Toronto fueron también espacios significativos en este sentido.

Con respecto al ensamblaje de Frankfurt, The authoritarian personality (1950) es una publicación que suele ser atribuida en exclusividad a Adorno, a pesar de que hay hasta cinco autores y autoras más: Else Frenkel-Brunswik, Daniel Levinson, Nevitt Sanford, Betty Aron y Mary Hertz Levinson. Estas dos últimas aparecen de nuevo bajo la expresión eufemística «en colaboración». Relata Fleck (2011: 261) las tensiones en torno a la autoría, que finalmente colocarían en primer lugar a Adorno, cuando el libro está basado en el trabajo de campo integrado por entrevistas llevadas a cabo por el Berkeley Public Opinion Study (liderado por Nevitt Sanford y Else Frenkel-Brunswik) y en las contribuciones previas de Else Frenkel-Brunswik sobre los tipos de personalidad. Frenkel-Brunswik, antes de formar parte del equipo que escribiría The authoritarian personality, contaba ya con una amplísima experiencia empírica y teórica en la medición de la personalidad y en cómo las personas respondían a la ambigüedad (Frenkel-Brunswik, 1939, 1940, 1948 y 1949). Este bagaje fue fundamental para articular la obra que injustamente se atribuye en exclusividad a Adorno, a pesar de que su contribución fue menor que la de Frenkel-Brunswik. Además, tal y como recoge Fleck (2011: 267-268), el análisis de la producción de este libro arroja que Frenkel-Brunswik escribió el $21 \%$, mientras que Adorno estuvo a cargo del $19 \%$.

Como vemos, esta publicación, que desarrolla cómo gente ordinaria puede terminar siendo fascista, arroja un nuevo nombre significativo en el ensamblaje de Frankfurt: Else Frenkel-Brunswik, quien se formó en el Círculo de Viena, al igual que hiciera Herta Herzog, un espacio geográfico interesante para el rastreo de las aportaciones femeninas en las décadas de 1920 y 1930 (Valsiner y Abbey, 2006: 10). Su tesis doctoral estuvo dirigida por Karl Bühler, quien también fuera director de tesis de Karl Popper. De formación psicoanalítica y positivista, en 1938 emigró a California, donde comenzó su colaboración con la Universidad de California Berkeley (Valsiner y Abbey, 2006: 10). Durante su vida no le fue posible acceder a una tenure track position (una plaza permanente en la universidad), debido a que ya tenía esta categoría su marido, Egon Brunswik, y las leyes del momento no lo permitían. Cuando su marido se suicidó, la Universidad de California le ofreció esta posibilidad, que Else Frenkel-Brunswik rechazó para acabar quitándose la vida ella también. 
Por otra parte, y en relación con la economía política y la Escuela de Chicago, es preciso destacar otros dos nombres: Mae Huettig y Helen Hughes, respectivamente. La primera de ellas, Mae Huettig, no ha sido parte del canon en crítica marxista, a pesar de que su tesis doctoral, Economic control of the motion picture industry: A study in industrial organization (1944), publicada por la University of Pennsylvania Press, fue el primer análisis del cine como industria desde una perspectiva económica (Phillips, 2015), tesis que fue un texto fundamental para Dallas Smythe, él sí, referente en economía política. Se hicieron eco del trabajo igualmente autores y autoras de referencia en el siglo XX, como Ball-Rokeach, Garnham o Wasko (Dorsten, 2012: 28 y 44). Por qué Huettig no ha formado parte del canon de economía política de los medios de comunicación es una pregunta difícil de entender y responder.

El trabajo de Helen Hughes, discípula de Robert E. Park, realizado en su tesis doctoral, News and the human interest story (Hughes, 1940), ha pasado desapercibido, a pesar de que se trata de una propuesta teórica interdisciplinaria ambiciosa a horcajadas entre el pensamiento crítico y el interpretativo, y es la primera historia y crítica de la función social y política de las noticias. $\mathrm{Ha}$ sido considerada una contribución única al conocimiento de la industria de la comunicación (Peters y Simonson, 2004). Hughes (1973) nos dejó un testimonio de enorme valor para conocer la intrahistoria femenina sobre el escaso reconocimiento y las vicisitudes vividas por las académicas esposas de profesores en los campus universitarios, una situación que ella reconoce fruto del sexismo y que la llevó a aceptar puestos menores como el de asistente editorial en el American Journal of Sociology (puesto que le ofreció Blumer, figura clave del interaccionismo simbólico). Su coautoría junto con Everett Hughes de la obra de referencia en el análisis racial, Where peoples meet: Racial and ethnic frontiers (1952), ha quedado también un tanto desdibujada, como ya hemos visto con otras publicaciones en las que participaron mujeres investigadoras.

Cierro este apartado con la mención a dos figuras femeninas en los estudios culturales y el determinismo tecnológico: Rachel Powell y Mary Q. Innis, respectivamente.

De Rachel Powell, investigadora asociada a tiempo completo en el Center for Contemporary Cultural Studies (CCCS), sabemos que escribió el primer artículo (denominado working paper) del CCCS de la Universidad de Birmingham: «Posibilities for local radio» (Powell, 1965), lo que marcó la rutina del centro y fue un importante primer paso editorial (Hoggart y Hall, 1965). Los denominados Working Papers in Cultural Studies, iniciativa editorial a modo de journal, fue un tipo de literatura gris fundamental para desarrollar las ideas intelectuales de esta nueva corriente que empezaba a formarse en la década de 1960. "Posibilities for local radio» es una publicación olvidada que, según Striphas y Hayward (2013: 104), debiera formar parte de los cuatro grandes: The uses of literacy (Hoggart, 1957); Culture and society (1780-1950) (publicado en 1958) y The long revolution (que vio la luz en 1961), ambos de Williams, y The making of the English working class (Thompson, 1963). Muy activa y prolífica en el centro, Powell criticó la condescendencia con la que 
era tratada por Stuart Hall, con quien disentía, en parte, por la omnipresencia de Hall y porque "eran los hombres los que marcaban la agenda" (Connell y Hilton, 2015: 303). Tras ella vendrían otras mujeres ya de la segunda generación, quienes, ellas sí, han tenido una presencia más importante, como Charlotte Brundson, Angela McRobbie o Dorothy Hobson.

Por último, destaca el rol que desempeńó Mary Q. Innis en el marco del determinismo tecnológico (Black, 2003; Belisle y Mitchell, 2018). Se formó en la Universidad de Chicago y se doctoró en la de Waterloo en 1965. Decana del College of Women (Universidad de Toronto) desde 1955 hasta 1964 (Scott, 2013) e hija de un ingeniero de telefonía, escribió sobre medios y comunicación antes que su marido, Harold Innis, fundador del determinismo tecnológico. Mary Q. Innis «transcribió, editó, escribió, investigó, preparó los índices e hizo de curadora de contenidos» (Belisle y Mitchell, 2018: 1) de las publicaciones de Harold, entre las que se encuentran los clásicos The fur trade (1927), The cod fisheries (1938) y Empire and communications (1950), dando forma a la lectura que hoy hacemos de él (Black, 2003: 433).

Aunque las pocas menciones que se hacen a su figura en las biografías de Harold Innis la presentan como una madre y una esposa devota, lo cierto es que, en palabras de su hija Anne Innis Dagg (Black, 2003: 438), «a ella le molestaba que la tomaran como una esposa de profesor universitario, cuando ella pensaba sobre sí misma como autora e investigadora por derecho propio».

El libro de Mary Q. Innis An economic history of Canada (Innis, 1935) fue la primera historia económica del país norteamericano y la evidencia más temprana de un escrito Innis sobre medios de forma explícita y amplia: incluye desde los servicios postales hasta los de telegrafía, pasando por los periódicos y la publicidad, además de la relación entre el transporte y la comunicación (Black, 2003: 433). El interés de Harold Innis por los medios de difusión y la comunicación no llegaría hasta comienzos de la década de 1940, con la publicación en 1942 de su artículo titulado «The newspaper in economic development» (Creighton, 1957).

\section{Conclusiones}

Las mujeres incluidas en este artículo estuvieron y vivieron, pero no han conseguido habitar y permanecer en la memoria colectiva de la disciplina. «Vivir sin habitar», "estar sin permanecer» son los lemas que describen la ausencia de académicas en el acervo y los relatos que conforman el campo de la comunicación. Esto ha sido así debido a que, a veces, hemos olvidado que el avance científico, también en comunicación, no es el resultado de mentes geniales, de héroes providenciales y aislados del contexto social y académico del momento, sino la consecuencia del esfuerzo colectivo. Un esfuerzo colectivo en el que también colaboraron las investigadoras, aunque, con frecuencia, hayan quedado fuera sus contribuciones, no se haya reconocido su participación activa y decisiva en los desarrollos teóricos y metodológicos o, directamente, se hayan atribuido sus aportaciones de manera injusta a colegas masculinos. 
Esta circunstancia no pasó desapercibida para algunas de estas académicas, de las que han quedado testimonios que recogen la frustración con la que vivieron ser «ciudadanas académicas de segunda clase», en palabras de Helen Hughes; ser consideradas solamente como madres y esposas, como hemos visto en el caso de Mary Q. Innis; la imposibilidad de acceder a una plaza estable en la universidad, como le pasó a Else Frenkel-Brunswik; la incomodidad de soportar actitudes paternalistas en los centros de investigación, tal y como lo vivió Rachel Powell, o la impotencia de no ser escuchadas, de vivir el aislamiento universitario y de ver cómo se imponía un paradigma, el positivista, que no podía responder a la complejidad de la comunicación de masas, tal y como le sucedió a Hortense Powdermarker.

¿Hubiera sido el campo, de manera oficial, más innovador, interdisciplinario e inclusivo si se hubiera dado una mayor visibilidad al acervo femenino y a sus autoras? Seguro, pues estos nombres apostaron ya en su tiempo por el eclecticismo teórico y la triangulación metodológica. Su visibilidad hubiera, además, promovido una disciplina más igualitaria y heterogénea.

Para tratar de compensar estos desequilibrios, he desarrollado un mapa de autoras alternativo al canon hegemónico con el que llevamos relatando la historia de nuestra investigación y enseñando teorías de la comunicación, al menos durante los últimos cincuenta años (a partir de la historia que Schramm publica a comienzos de la década de 1960).

Los paradigmas no cambian por sí solos, de ahí la necesidad de seguir implementando una epistemología crítica que contribuya a facilitar la integración de los aportes y el equilibrio entre todos los actores científicos. Recuperar la memoria de mujeres investigadoras es una forma de restituir la injusticia académica y social que sufrieron ellas y que arrastramos y sufrimos todas las personas que hoy habitamos y enseñamos las teorías de la comunicación. Porque borrándolas a ellas, «nos borramos» también a nosotros mismos.

\section{Referencias bibliográficas}

Adorno, T.; Frenkel-BrunswiK, E.; LeVInson, D. y SANFord, N. (1950). The authoritarian personality. Nueva York: Harper.

ASHCRAFT, K. y SiMONSON, P. (2015). "Gender, work and the history of communication research». En: SIMONSON, P. y PARK, D. (coords.). The international history of communication study. Nueva York: Routledge, 47-68.

Belisle, D. y MitCHell, K. (2018). «Mary Quayle Innis: Faculty wives' contributions and the making of academic celebrity». The Canadian History Review, 99 (3), 456-486. <https://doi.org/10.3138/chr.2017-0108>

Berelson, B.; Lazarsfeld, P. y MCPheE, W. (1954). Voting. Chicago: The University of Chicago Press.

BLACK, D. (2003). "Both of us can move mountains: Mary Quayle Innis and her relationship to Harold Innis' legacy». Canadian Journal of Communication, 28 (4), 433-447. <https://doi.org/10.22230/cjc.2003v28n4a1391> 
BRUNSDON, Ch. (2000). The feminist, the housewife, and the soap opera. Oxford: Clarendon Press.

BURTON, D. y DARCY, R. (1985). "Careers of men and women in the profession». Western Political Quarterly, 38 (1), 132-147. <https://doi.org/10.1177/106591298503800110>

Cantril, H.; Gaudet, H. y Herzog, H. (1940). The invasion from mars. Nueva Jersey: Princeton University Press.

COMmire, A. y KlezMer, D. (2007). «Frenkel-Brunswik, Else (1908-1958)». Dictionary of Women Worldwide: 25,000 Women through the Ages, 1, 694.

Connell, K. y Hilton, M. (2015). «The working practices of Birmingham's Centre for Contemporary Cultural Studies». Social History, 40 (3), 287-311. <https://doi.org/10.1080/03071022.2015.1043191>

Craig, R. (1999). "Communication theory as a field». Communication Theory, 9 (2), 119-161. <https://doi.org/10.1111/j.1468-2885.1999.tb00355.x>

Creighton, D. (1957). Harold Adams Innis. Toronto: University of Toronto Press.

Dorsten, A. (2012). "Thinking dirty: Digging up three founding matriarchs of communication studies». Communication Theory, 22 (1), 25-47. <https://doi.org/10.1111/j.1468-2885.2011.01398.x>

- (2016). "Women in communication research». En POOLEY, J.; JenSEN, K. y CRAIG, R. (coords.). The international encyclopedia of communication theory and philosophy. Nueva Jersey: Wiley, 1-13. <https://doi.org/10.1002/9781118766804.wbiect106>

Douglas, S. (2006). "Personal influence and the bracketing of women's history». The Annals of the American Academy, 608, 41-50. <https://doi.org/10.1177/0002716206292458>

FLECK, C. (2011). A transatlantic history of the social sciences. Londres: Bloomsbury Academic.

FRENKEL-BRUNSWIK, E. (1939). «Mechanisms of self-deception». Journal of Social Psychology, 10, 409-420. <https://doi.org/10.1080/00224545.1939.9713377>

- (1940). "Psychoanalysis and personality research». The Journal of Abnormal and Social Psychology, 35 (2), 176-197. <https://doi.org/10.1037/h0060754>

— (1948). "A study of prejudice in children». Human Relations, 1, 295-306. <https://doi.org/10.1177/001872674800100301>

- (1949). "Intolerance of ambiguity as an emotional and perceptual personality variable». Journal of Personality, 18, 108-143. <https://doi.org/10.1111/j.1467-6494.1949.tb01236.x>

García-JiméneZ, L. (2019). En defensa de la comunicación. Valencia: Tirant lo Blanch.

García, F.; Zurián, F. y NúŃEZ, P. (2020). «Los estudios de género en los grados de comunicación». Comunicar, 63, 21-30. <https://doi.org/10.3916/C63-2020-02>

HiER, S. y KEMP, C. (2002). «Anthropological stranger: The intellectual trajectory of Hortense Powdermaker». Women's History Review, 11 (2), 253-272. <https://doi.org/10.1080/09612020200200320>

HogGaRT, R. (1957). The uses of literacy. Aspects of working-class life. Londres: Chatto $\&$ Windus. 
HoggarT, R. y Hall, S. (1965). «Introduction». En: POWELL, R. «Possibilities for local radio». Occasional Paper, 1, 1-22.

HuetTig, M. D. (1944). Economic control of the motion picture industry. Filadelfia: University of Pennsylvania Press.

HugHes, H. (1940). News and the human interest story. Chicago: University of Chicago Press.

- (1973). «Maid of all work or departmental sister-in-law?: The faculty wife employed on campus». American Journal of Sociology, 78 (4), 767-772. Recuperado de $<$ https://www.jstor.org/stable/2776602>

INNIS, M. Q. (1935). An economic history of Canada. Toronto: Ryerson.

JANSEN, S. (1993). "The future is not what it used to be: Gender, history, and communication studies». Communication Theory, 3 (2), 136-148. <https://doi.org/10.1111/j.1468-2885.1993.tb00063.x>

KatZ, E. y LAZARSFeld, P. (1955). Personal influence. Nueva York: The Free Press.

Klaus, E. y Seethaler, J. (2016). What do we really know about Herta Herzog? Frankfurt: Peter Lang.

Lazarsfeld, P.; Berelson, B. y Gaudet, H. (1944). The People's choice. Nueva York: Columbia University Press.

Merton, R.; Fiske, M. y CURTIS, A. (1946). Mass persuasion. Nueva York: Harper.

Merton, R.; Fiske, M. y Kendall, P. (1948). The focused interview. Nueva York: Free Press.

MEYEN, M. (2012). "The founding parents of communication». International Journal of Communication, 6, 1451-1459.

PARK, D. y POOLEY, J. (coords.) (2008). The History of Media and Communication Research: Contested Memories. Nueva York: Peter Lang.

Peters, J. y SimOnSON, P. (2004). Mass communication and American social thought: Key Texts, 1919-1968. Oxford: Rowman.

PHILlIPS, W. (2015). "A Maze of intricate relationships: Mae D. Huettig and early forays into film industry studies». Film History, 27 (1), 135-163. <https://doi.org/10.2979/filmhistory.27.1.135>

PoOlEY, J. (2008). «The new history of mass communication research». En: PARK, D. y POOLEY, J. (coords.). The history of media and communication research: Contested memories. Nueva York: Peter Lang, 43-70.

- (2017). "Wilbur Schramm and the "four founders" history of U.S. communication research». Коммуникации Медиа Дизайн, 2 (4), 5-18. $<$ http://dx.doi.org/10.17613/M6Q859>

POWELL, R. (1965). "Possibilities for local radio». Occasional Paper, 1, 1-22.

Rodrigo Alsina, M. (2001). Teorias de la comunicación: Ámbitos, métodos y perspectivas. Barcelona: Aldea Global.

ROGERS, E. (1994). A history of communication study: A biographical approach. Nueva York: Free Press.

ROWland, A. y SimOnSON, P. (2013). "The founding mothers of communication research: Toward a history of a gendered assemblage». Critical Studies in Media Communication, 31 (1), 3-26. <https://doi.org/10.1080/15295036.2013.849355>

SCHRAMM, W. (1963). "Communication research in the United States». En: SCHRAMM. W. (coord.). The science of human communication. Nueva York: Basic Books, 1-16.

ScOTT, M. (2013). «Mary Quayle Innis». The Canadian Encyclopedia. 
SIGNORIELLI, N. (1996). Women in communication: A biographical sourcebook. Westport: Greenwood Press.

SimONSON, P. (2012). "Mail and females at the bureau: The happiness game in the gendered contexts of early U.S. communications research». International Journal of Communication, 6, 1277-1289.

- (2016). "Herta Herzog and the founding mothers of mass communication research». En KLAUS, E. y SEETHALER, J. (coords.). What do we really know about Herta Herzog? Nueva York: Peter Lang, 61-84.

SimONSON, P. y ARCHER, L. (2011). «Women in media research». Documental Out of the Question.

SIMONSON, P. y PARK, D. (coords.) (2015). The international history of communication study. Nueva York: Routledge.

STRIPHAS, T. y HAYWARD, M. (2013). «Working papers in cultural studies, or, the virtues of grey literature». New Formations: A Journal of Culture, 78, 102-116. <https://doi.org/10.3898/NewF.78.05.2013>

THOMPSON, E. P. (1963). The making of the English working class. Harmondsworth: Penguin.

VALSINER, J. y ABBEY, E. (2006). «Ambivalence in focus: Remembering the life and work of Else Frenkel-Brunswik». Studies in Psychology, 27 (1), 9-17. <https://doi.org/10.1174/021093906776173126>

Vera BALANZA, T. (2012). «Periféricas: Propuesta didáctica para teorías de la comunicación». $Z E R, 32,13-27$.

VORDERER, P.; PARK, D. y LUTZ, S. (2019). "A history of media effects research traditions». En: Oliver, M.; RANEY, A. y BRYANT, J. (coords.). Media effects: Advances in theory and research. Nueva York: Routledge, 1-15.

WiLLIAMS, R. (1958). Culture and society (1780-1950). Londres: Chatto \& Windus.

- (1961). The long revolution. Londres: Chatto \& Windus. 
